XI.-Solution and Pseudo-solution. Part I.

By Harold Picton and S. Ernest Linder.

INTRODUCTION.

Is the previous paper, one of us has examined three special cases u apparent solution, namely, those of mercury, antimony, and arsenic sulphides. These liquids, which in many ways resemble ordinary colloidal solutions, were found to present a series passing from matter in a state of subdivision not too fine to allow of its observation under the microscope, to particles so fine as to diffuse in the manner of ordinary solutions. Arsenic sulphide, indced, was found capable in itself of presenting us with three diffcrent types of solution, distinguished in the paper as arsenious sulphide $\alpha, \beta$, and $\%$. Arsenic $\alpha$ is made up of particles just visible under the microscope, arsenic $\beta$ contains no visible particles, whilst arsenic $\%$ contains particles so minute as to be diffusible. The case of mercury sulphide was specially interesting, as the sulphide, after precipitation, is easily dissolved in sulphuretted hydrogen water, and in this solution the vibrating particles are clearly discernible under the microscope (see previous paper).

We have here what appears to be a half-way stage towards true solution. The suggestion is at once forced upon our minds that the state of pseudo-solution would gradually merge into that of true solution by carrying the subdivision farther and farther, till finally, perhaps, we may have the substance dissociated into ions. To trace the steps somewhat further would obviously be a task of no little interest. And on asking ourselves what solutions are most likely to furnish stages in the phenomena of solution between those of arsenic $\gamma$ and those of true solution, the case of ordinary colloidal solutions at once suggests itself. Thus the investigation into the nature of the sulphide solutions just described had not proceeded far before it was determined to follow it up with some experiments on a variety of colloïdal solutions. Some of these have now been examined, and we have also obtained interesting evidence with regard to the transition from colloidal to crystalloidal solution, part of which we shall lay before the Society in this paper.

Before describing our own experiments, it will be well to give a short account of previous work on the subject.

Graham, it will be remembered, supposed the non-dialysability of colloids to be due to the size of their molecules, which be considered too large to pass through the pures of the membrane. A certain mystery has long hung about the facts of colloidal solution, 
and that colloids are substances of very great molecular complexity has of late become increasingly evident. Theory has oscillated between the two conceptions of very large molecular aggregates and very finely divided particles. Perhaps in the end the two theories mean the same thing.

Ostwald (Lehrb. d. allg. Chem., 1, 52) and Paterno (Zeit. physikal. Chem., 4, 457) have both suggested that colloidal solutions are probably composed of very finely divided solids in a state of suspension. But experimental evidence has been almost entirely wanting.

Barus and Schneider, in a paper on colloidal silver in the Zeitschrift für physikalische Chemie $(8,278)$, have recently made an attempt to supply this want. They support the theory that colloidal solution in general is merely a condition of suspension.* With the main tendency of their arguments we are in agreement, but there are some portions of their work to which we are obliged to take exception. We propose to briefly examine the paper here. Barus and Schneider have specially investigated the case of Carey Lea's soluble silver. Their solutions were obtained by reducing with ferrous citrate, and redissolving the silver thus obtained. The solution presents many points of similarity to the sulphide solutions discussed by one of us in the previous paper. It is slightly fluorescent, it is coagulated by the addition of certain substances, and also by freezing, but it does not settle spontaneously. The solution, however, does not lend itself so easily to exact chemical or physical examination, since it cannot be obtained free from iron salts.

The experimental work of the paper may be considered under two heads : first, experiments on the electrical resistance of the silver solution; and, secondly, experiments on the sedimentation of the solution when partially coagulated. The silver in solution is found to act as a non-conductor ; indeed, according to the experiments, the stronger the silver solution the greater the resistance. This is somewhat unintelligible, but the measurements are complicated by the presence of electrolytes in the solution. From the fact that the metallic silver in solution does not act as a conductor, they conclude that it is not present in the molecular state, but rather in the condition of finely-divided suspended particles. This reasoning appears, however, to us to be by no means conclusive, for what evidence is there that would lead us to expect silver in a state of true solution to act as a conductor?

The coagulation of pseudo-solutions, on the addition of certain salts or acids, cannot be ascribed merely to diminished viscosity of the

* To prevent any misconception, we may say that our work on the sulphides (see preceding papers) was begun in 1888 , and that the present paper was actually being prepared before we became aware of this work.

VOL. LXI. 
water, since the traces of acid or salt necessary would be quite inadequate to produce so profound an effect. This had previously been shown by Barus. The authors suppose the coagulation to be due to the separation of the particles.

To arrive at some idea of the size of the silver particles, the authors make use of the following equation:-

$$
x=\frac{2}{9 \eta} r^{2}\left(\rho-\rho^{\prime}\right) g,
$$

where

$x=$ rate of subsidence of the particle.

$\eta=$ viscosity of the suspending medium.

$\rho=$ specific gravity of particle.

$\rho^{\prime}=$ specific gravity of medium.

$r=$ radius of particle.

Now $x, \eta, \rho$, and $\rho^{\prime}$ are constants, $g$ is obviously constant, and thus $r$ alone varies.

As the silver particles do not subside, they have to base any calculations on a partially coagulated solution. Inserting values in the above equation, and taking Kohlrausch's value for the size of the water molecule, they find that the diameter of the particles in a coagulating silver solution may be as small as that of 70 water molecules. Calculations of this kind are very fascinating, but we confess to grave doubts as to the validity of the above equation. It is evident from the fact that the solvent can effect this fine subdivision, that there is some action between solid and solvent, whilst the above equation presupposes none. The fact, too, that particles visible under the microscope exhibit so very little tendency to settle does not seem to coincide with the assumptions of the equation.*

Prange is quoted as having observed evolution of heat on coagulation of the silver solution. Prange is also quoted as having obtained a negative result with Tyndall's experiment (Kec. d. Trav. Chim. des Pays-bas, 9, 125).

The evidence upon which most stress is laid in the above-quoted paper seems to us to be decidedly inconclusive. It is also noteworthy that throughout this paper there is an assumption that if the colloidal solutions contain very finely divided particles, the solution is a fact of mere mechanical suspension and nothing more. This is evident, too, from their method of calculating the size of the suspended particles. An assumption of this kind seems to us to be quite irreconcilable with fact. There is a reaction other than mechanical between the solvent and the solid, even in these cases of colloidal solution. Otherwise the re-

* Compare some observations of Jackson (Proc. Chem. Soc., 1891, 178) mentioned in the discussion of this paper. 
solubility of precipitated colloids becomes an impossibility, and the extreme permanence of some colloidal solutions is exceedingly hard to explain. Moreover it will be shown, in at least one case in the following paper, that there is undoubted molecular attraction or chemical combination between the colloid and its solvent.

\section{General Statement.}

Our intention in this part of the paper is to carry further the examination, begun by one of us, into the connection between suspension and solution. We have now made a general survey of the ground, and we propose in this section to trace further the change from obvious suspension to true colloidal solution, and to make some attempt to follow the apparently continuous change into the regions of crystalloidal solution. Our object being to follow this continuous change, rather than to examine individual cases, we have embodied in the present part results obtained with typical examples, and we shall leave the examination of a larger array of individual cases for a future occasion.

Whatever can throw any light upon the mechanism of solution must be of interest. We have undertaken to follow up the various states of suspension and solution through their different grades, in the hope that an exact examination of the changes which occur in the passage from one grade to another may shed some light upon the vexed question, What is it that happens when a body dissolves?

The questions to which we seek an answer in these researches are, broadly, the following:-

What is the nature of colloidal solution? Does the solid, dissolved in these solutions, exist in the form of finely-divided particles, discernible by optical or other means? If such be the constitution of any colloidal solution, would it in any case be possible to see these particles under the microscope? Does the dissolved substance in any crystalloidal solution exist in the form of particles which can, by any means, be discerned? May there be a perfectly continuous gradation from suspension to crystalloidal solution? Supposing that such a series can be made out, what is the nature of the forces by which, in the lower grades of solution, the particles are held in suspension? What is the nature of these finely-divided particles, if they exist?

In the foilowing pages we hope to show how these questions can be answered.

\section{Experimentar Results.}

The colloids at present examined have confirmed the suspicions suggested by the investigation of the sulphide solutions. The present 
paper only deals with the few cases at present examined, and does not pretend to be in any way exhaustive. The results of the more extended investigation which we intend to undertake, both of colloidal solution, and, if possible, of its passage into crystalloidal solution, we shall embody in Part II of this paper, which we hope to be able before long to lay before the Society. In the present part, we shall set down briefly the results obtained with the solutions already examined. Of inorganic colloids, the following have been examined:-

\section{Ferric Hydrate.}

The hydrate was precipitated with ammonia from a solution of ferric chloride, well washed, and then placed in a solution of ferric chloride, in which it soon dissolved. Without dialysing out the excess of ferric chloride, some of this was examined, whilst another portion was dialysed till the outer water contained no trace of chloride.

The solution was, in both cases, fluorescent, but absolutely clear by transmitted light.

Microscopical Examination.-A magnifying power of 1000 diameters was found quite inadequate to reveal any want of homogeneity in the structure of the solution.

Tyndall's Experiment.-A tube of the undialysed liquid was sealed and examined with a beam of light, in the same manner as thesulphide solutions (see p. 143). A dense glow was observed in the track of the beam, the light of which was completely polarised. To confirm this result, a portion of the liquid which had been examined was treated with hydrochloric acid till the hydrate was entirely dissolved. The track of light was no longer visible, with the exception of the very feeble beam always observed, even with distilled water. The dialysed solution showed the track of the beam so markedly that it was only necessary to hold the tube under an ordinary gas jet to observe the effect.

Filtration through a Porous Cell.-Ferric chloride solution having. been saturated with the hydrate, the liquid was dialysed. The ferric chloride was evidently, for the most part, combined with the hydrate, for only slight diffusion was observed. Dialysis was continued till the outer liquid showed no trace of iron or of chloride. The dialysed liquid had, by transmitted light, a clear, deep, ruby-red colour. It was filtered, under pressure, through a porous cell. The filtrate contained no trace of either iron or chloride. Evidently, therefore, any remaining ferric chloride was held in combination by the hydrate. None of the compound is present in a suficiently finely-divided state to pass through a porous cell. 
Coagulation Experiment.-The solution is easily coagulable by calcium chloride. On coagulation, the whole of the iron passes out of solution, the filtered liquid containing no iron. The combined molecules of hydrate and chloride coagulate without dissociation. It is thus quite clear that, in this case, there is a chemical attraction between the molecules of the solvent (ferric chloride) and the solid (ferric hydrate).

The act of coagulation is not accompanied by any evolution of heat. The molecules, or particles, are too large to produce perceptible evolution of heat on condensation.

Diffusion Experiment.-Some of the dialysed hydrate was set diffusing, without a membrane, on a Saturday. By the following Monday no diffusion had occurred. This places the state of subdivision at a probably lower stage than that of the diffusible arsenic sulphide. But, on the other hand, the result might, perhaps, be due to slower rate of vibration without increase of size.

Crystallisalility of the Solution.-It is stated that a strong solution of ferric hydrate in ferric chloride, when evaporated in a vacuum, deposits crystalline plates of the compound $9 \mathrm{Fe}_{3} \mathrm{O}_{3}, \mathrm{FeCl}_{3} \cdot{ }^{*}$ If this be so, we have the remarkable fact of crystallisation in a body, the molecular aggregates of which are sufficiently large to scatter light, as shown by Tyndall's experiment. The proportion of chloride present, according to this formula, being, probably, larger than that in our solution, a considerable excess of ferric chloride was added to some of the colloidal hydrate, with a view to determine any change in the character of the solution. Immediately after the addition, it was examined with a beam of light. The same appearance was noticed as in the case of the original hydrate. After two or three days had elapsed, it was again examined. Just the same luminous beam was still observed.

\section{Chromic Chloride.}

While engaged in preparing some colloidal chromium hydrate, we had occasion to notice certain facts with regard to the solubility of chromium chloride. As is well known, prolonged boiling with water will only effect gradual dissolution of chromium chloride. The addition of a trace of chromous chloride, however, makes it dissolve much more rapidly. In our case, some beautifully crystalline "resublimed" chromic chloride was treated with water, and a littlo chromous acetate, containing excess of hydrogen chloride, then added. In the cold, dissolution was slowly effected. Some of the liquid was allowed to stand for some time, and then sealed in a glass tube.

* This formula is taken from Ramsay's System of Inorganic Chemistry. 
Tyndall's Experiment.-The liquid was examined in the same way as before, and was found to give a well-marked, soft beam. We concluded that the chromic chloride was probably dusty or impure. After keeping it a few days, the tube was again examined, and this time the track of the beam of light was no longer visible. The hydrochloric acid present would prevent the formation of basic salts. From the results of our other experiments, it seems to us most probable that we are here actually observing the slow breaking up of molecular aggregates with the final formation of a crystallisable solution. The theoretical meaning of these experiments will be discussed further in the sequel.

\section{Chromic Hydrate.}

The solution of chromic hydrate in chromic chloride shows a wellmarked, luminous beam. It was, therefore, needless to examine the dialysed solution. The chromic chloride used showed no luminous beam.

\section{Aluminium Hydrate.}

The solution of aluminium hydrate in the chloride showed a wellmarked, luminous beam.

Both these solutions are thus similar to the ferric hydrate solution.

\section{Silicic Acid.}

Silicic acid affords a very characteristic case of colloïdal solution, and it is thus of obvious interest to examine it. Sodium silicate solution was treated rapidly with excess of hydrochloric acid so as to redissolve the silicic acid formed.

\section{Solution in Presence of Hydrochloric Acid.}

Tyndall's Experiment.-The undialysed solution, in presence of a very slight excess of hydrochloric acid, showed no luminous berm on passing a ray of light through the liquid. Some of the dialysed solution, to which hydrochloric acid had been added, showed no luminous beam. Some of the silicic acid solution, in presence of hydrochloric acid, was gelatinised by evaporation and redissolved in presence of acid. It showed no luminous beam. It is, therefore, evident that, in presence of hydrochloric acid, silicic acid does not in solution form molecular aggregates of sufficient size to scatter light.

Evaporation.-As the solution did not scatter light, it was thought that if evaporated in presence of hydrochloric acid it might possibly crystallise. Some of it was allowed to slowly evaporate in a current of dry air mixed with hydrochloric acid. It gelatinised, however, 
thus seeming to behave like a colloid, though too finely subdivided to yield a positive result with Tyndall's experiment.

Filtration through a Porous Cell.-A strong solution of silicic acid was obtained by the rapid addition of hydrochloric acid to sodium silicate. The solution was then filtered as before through a porous cell. Silicic acid at once came through in large quantities. It was obtained in the amorphous state after evaporation on the water-bath and dissolving out the sodium chloride. This result is particularly interesting as presenting us with the case of a colloid the molecules of which can pass through the fine pores of a cell. The cases of ferric hydrate and hæmoglobin are in interesting contrast to this.

\section{Dialysed Solution Free from Hydrochloric Acid.}

The solution was set dialysing on Wednesday. It was left, with frequent changes of water, till Saturday, and then without change till Monday. The solution was now free from chloride.

Tyndall's Experiment.-A portion of the solution was sealed up on Monday (October 19). Examined with lime light, it gave a very feebly luminous beam, scarcely greater than that observed with ordinary distilled water. On Tuesday, the tube was re-examined, and this time a distinct, soft glow was observed in the track of the light. Obviously, a slow condensation was taking place after the removal of the hydrochloric acid. From October 23 to November 23 no further change has been observed in the tube.* The silica, in presence of a very slight excess of acid (see above), continues to show no beam. A few filaments of silica have, however, developed in the tube. The other acid solutions (see above) are unchanged.

\section{Molybdic Acid.}

Some pure "colloidal molybdic acid" solution was kindly lent us by Miss Aston. The solution was allowed to stand in tubes for about 10 days, to allow some small, solid particles to settle. On evaporation on the water-bath, the solution leaves a gelatinous film which breaks up into radiating rods, giving it an almost crystalline appearance. Evaporated over sulphuric acid, a gelatinous residue is also obtained.

Tyndall's Experiment.-Only a very faint track of light, like that observable with distilled water, could be seen on passing a ray of light through this liquid. The solution does not, therefore, contain molecular aggregates large enough to possess the power of scattering light.

* The silica solution was dilute. 
Filtration through a Porous Cell.-The molybdic acid passes at once through our porous cell.

The following organic substances have been examined :-

\section{Cellulose.}

Cellulose was dissolved in Schweitzer's solution, and the clear liquid allowed to settle for several days. The Schweitzer's solution itself showed a very feeble luminous beam when a ray of light was passed through it.

Tyndall's Experiment.-The cellulose solution showed a well marked glow when the ray of light was passed through it. The light of this glow was, as usual, polarised.

\section{Starch.}

Soluble starch was prepared by dissolving starch in glycerine, pouring into water, and then precipitating by alcohol. The precipitated starch was redissolved in water and again precipitated. A strong solution was made from the starch so obtained.

Tyndall's Experiment.-A tube of the clear solution showed a wellmarked, luminous beam with a ray of light. Polarisation was com. plete.

Diffusion Experiment.-A strong starch solution was set diffusing on Wednesday, July 8. By a siphon, fixed to the side of the beaker, samples of a diffusate could be drawn off without causing any disturbance in the liquid. On Wednesday, July 15, samples of the outer liquid.were drawn off. This gave so faint a colour with iodine that, in a test-tube, the tint was hardly perceptible. Practically, no diffusion had occurred. It is no use exposing the starch solution to more prolonged diffusion, as it undergoes chemical changes, and the experiments are thus invalidated. Some of the starch solution from the diffusing bottle was tested with a ray of light, and gave a wellmarked, polarised beam.

From these experiments, it appears probable that the starch exists in solution in states of aggregation larger than those of a diffusible arsenic solution.

\section{"Congo-Red."}

In some experiments on the filterability of bodies of varying molecular complexity through porous pots, we made use of Congo-red, $\mathrm{C}_{6} \mathrm{H}_{4} \cdot \mathrm{N}: \mathrm{N} \cdot \mathrm{C}_{10} \mathrm{H}_{5}\left(\mathrm{NH}_{2}\right) \cdot \mathrm{SO}_{3} \mathrm{Na}$. $\mathrm{C}_{6} \mathrm{H}_{4} \cdot \mathrm{N}: \mathrm{N} \cdot \mathrm{C}_{10} \mathrm{H}_{5}\left(\mathrm{NH}_{2}\right) \cdot \mathrm{SO}_{3} \mathrm{Na}$. Though not of service for the purposes we at first intended, it has yielded, among others :- 


\section{Neutral Solution.}

Congo-red dissolves very easily in water, giving a deep, red-brown solution.

Tyndall's Experiment.-The fluorescent solution gives a well-marked, polarised beam.

Filtration through a Porous Cell.-The very deep-coloured solution was filtered under pressure for nearly two hours. At least 60 c.c. of water were passed through the small porous pot. The inner liquid showed only a faint tinge of colour, probably due to some slight leak.

Diffusion Experiment.-In this experiment, a slight amount of coloration of the outer water was noticed during the first two days, but did not afterwards perceptibly increase. The coloration might be due to slight currents or to the presence of some diffusible substance. Certainly the Congo-red, as a whole, did not diffuse. The same solution was recovered on Friday, the outer water changed, and the diffusion allowed to proceed till Monday. A very slight coloration of the outer liquid was observed, less than in the previous case.

\section{Acid Solution.}

The acid Congo-red has a fine purple-blue colour.

Tyndall's Experiment.-With a ray of light, this gives a wellmarked, polarised beam.

\section{Alkaline Solution.}

The alkaline Congo-red has a clear, red colour without the brown tinge of the neutral substance.

Tyndall's Experiment.-This solution showed no luminous beam when a ray of light was passed through it.

Filtration through a Porous Cell.-Filtered under pressure, the filtrate was in 20 minutes as strongly coloured as the outer liquid.

\section{Homoglobin.}

In seeking for a body likely to be of service in connecting colloidal with crystalloidal solution, we naturally turned cur attention to the case of hamoglobin. This substance is easily obtained; it forms several readily crystallisable derivatives, and it affords an instance of enormous molecular complexity. Its percentage composition ( $\mathrm{dog}$ ) is given as:- $\mathrm{C}, 53.85 ; \mathrm{H}, 7.32 ; \mathrm{N}, 16.17 ; \mathrm{Fe}, 0.42 ; \mathrm{S}, 0.39 ; \mathrm{O}, 21.84$. Preyer assigns it the empirical formula $\mathrm{C}_{600} \mathrm{H}_{960} \mathrm{~N}_{154} \mathrm{FeS}_{3} \mathrm{O}_{179}$. It is possible that the molecular weight may be much greater than this. 
Owing to the small percentage of iron present, this is the simplest formula that can be assigned. Obviously it is of great interest to determine the analogies between a crystallisable solution containing these exceedingly complex molecules and the colloidal solutions, some at least of which we have seen contain aggregates large enough to scatier and polarise light.

\section{Oxyhacemoglobin.}

Preparation.-Fresh, defibrinated dog's blood was shaken with ether and allowed to stand till it had formed a magma of crystals The liquid was then centrifugalised, the upper layer drawn off, and the crystals well stirred with water and alcohol. The mixture was again centrifugalised, the washing being repeated five times. The crystals thus purified from serum-albumin were dissolvod in the smallest quantity of water, alcohol added, and the solution allowed to stand in ice. In the course of 24 hours, the hæmoglobin had again crystallised out. After once more centrifugalising, the hæmoglobin obtained was used in the following experiments. The red mass, when examined under the microscope, showed a beautifully crystalline structure.* On allowing some of these crystals to drop into water. they dissolve, especially if the water be very slightly warmed, forming a beautiful, clear, red solution.

Tyndall's Experiment.-Some of the red solution was filtered, a few floating particles being discernible. The perfectly clear liquid was then examined with a ray of lime light. A distinct, soft, luminous beam was observed, the light of which was completely polarised. The solution contained about 0.3 or 0.4 per cent. of oxyhæmoglobin, and it seems quite impossible that in a solution so dilute there could have been sufficient impurity to account for this beam. Besides this, presence of dust or other solid impurities is indicated by a beam of quite different appearance, and, if the expression may be permitted, by one far less close in texture. We have every reason to believe that the hæmoglobin solution thus carefully prepared contains no impurity sufficient to mar these results.

Examined spectroscopically, the solution gave the characteristic and well-marked absorption spectrum of oxyhæmoglobin.

We have here what is probably the second case (see ferric hydrate) of a crystallisable solution revealing the presence of molecular aggregates or molecules sufficiently large to scatter light.

Filtration through a Porous Cell.-This method seems to possess

* It is quite possible, with appropriate means, to obtain crystals several centimeters in length, but for our purpose the above method was sufficient. These crystals can be obtained from defibrinated blood without any addition of alcohol. 
considerable advantages over the ordinary one of dialysis. In the first place, it is difficult to obtain a thin membrane quite free from inequalities, and, in the next place, the process of dialysis is, necessarily, rather prolonged, and in the case of rather unstable bodies is, therefore, not very applicable. The crystallised hæmoglobin is said to be capable of diffusing very slowly through animal membranes. Owing to the rapid and decisive results obtained by the above method in other cases, we applied this test to the dissolved hæmoglobin crystals. The solution was, therefore, filtered under pressure in the way described in the previous paper. After filtering for 2 or 3 hours and thus obtaining three cellfuls of filtrate, the liquid still came through the cell as clear and colourless as pure distilled water, while the onter liquid had a brilliant scarlet hae. The hæmoglobin molecules or their aggregates are, therefore, too large to pass through the pores of our porous cell. As in the case of other solutions possessing this property, the molecular aggregates are large enough to scatter and polarise light.

These results make it impossible to avoid the conclusion that there is no hard and fast line between colloidal and crystalloidal solution. More strikingly does this seem to be the case when we remember that silicic acid, in presence of hydrochloric acid, passes readily through the pores of a porous cell, though it has not been obtained crystalline from this solution, and must, therefore, at least crystallise far less readily than hæmoglobin.

\section{Carbonic Oxide Hamoglobin.}

This derivative of hæmoglobin is crystalline and very stable. Stirling mentions that he has kept carbonic oxide hæmoglobin in a, stoppered bottle for four years without alteration (Landois and Stirling, 1, 34). It is readily obtained by passing carbonic oxide through a solution of bæmoglobin. The solution thus obtained from the recrystallised hæmoglobin was examined spectroscopically, and showed the characteristic shifting of the two oxyhæmoglobin bands towards the violet.

Tyndall's Experiment.-Examined with a ray of light, the solution showed a well-defined, luminous beam. A strong solution of the carbonic oxide hæmoglobin was recrystallised, and some of the crystals redissolved. The same beam was again observed, and the light was found to be completely polarised.

Filtration through a Porous Cell.-As in the case of oxyhæmoglobin, the solution could not pass through the pores of the cell. The filtrate was absolutely colourless. It is noteworthy that this observation coincides with the general rule, hitherto observed, that non- 
filterability is coincident with inability to scatter light. Exceptions to this rule may, however, be hereafter found.

\section{Magdala-Red. Naphthylamidoazonaphthalene $\left(\mathrm{C}_{30} \mathrm{H}_{20} \mathrm{~N}_{4}\right)$.}

This substance, being readily obtainable, was chosen as a crystalline body of less complexity than hæmoglobin, but still possessing a complicated molecule. Its vivid colour also made it peculiarly serviceable in our experiments (see below). The solution in alcohol is bright pink by transmitted light, and has a magnificent golden fluorescence.

Tyndall's Experiment.-The track of the ray of light was marked by a beautiful glow of colour. When examined with the Nicol's prism, the scattered light was found not to be polarised.

Filtration through a Porous Cell.-The alcoholic solution filtered through a porous cell at once showed the vivid colour of Magdala-red in the filtrate.

\section{A new Property of certain Solutions.}

We propose in this place to give some preliminary account of a remarkable property we have observed in some solutions; this consists in the repulsion of the dissolved substance as a whole from one pole to another when we immerse in the liquid electrodes connected with a galvanic battery. Our experiments are not, as yet, far advanced, but it seems appropriate in this place to give a preliminary account of them, as they may prove of service in ascertaining the

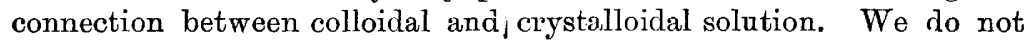
propose to do more here than give some of the experimental results obtained. It is difficult to frame a theory which will account for them, but the facts are as follows. We will give, first, the results obtained with arsenic sulphide, as this substance was most thoroughly investigated; and then some experiments dealing with substances ranging from pseudo-solution to crystallisable solution.

Arsenious Sulphide.-The first experiments consisted in immersing the electrodes connected with a storage battery in some of the diffusible arsenic sulphide solution. As was expected, no appreciable electrolysis occurred, but the sulphide was gradually driven down below the electrodes. It showed no signs of coagulation, but, on the contrary, when the connections were broken, it began to diffuse up slowly through the liquid. This is not an effect of decomposition, nor does gravity play more than a subsidiary part, as is shown by the subsequent experiment.

In the next experiment, a $\mathrm{V}$-tube was partially filled with the 
arsenic solution and the electrodes just immersed in the liquid. The electrodes were at a distance of about $30 \mathrm{~cm}$. from each other. In about 10 minutes, the sulphide was observed to be descending from the negative electrode. The descent slowly continued till about $6 \mathrm{~cm}$. of colourless liquid were observable at the negative pole. The liquid at the positive electrode was, meanwhile, unchanged, and remained so for an hour and a half, during which it was observed. The experiment was left all night, and by next morning a considerable descent of the liquid in the limb containing the positive. electrode was also observed. The colourless liquid at the negative pole, when tested, was found to contain neither arsenic nor sulphuretted hydrogen.

A much more definite experiment was made with the use of a tube of the kind shown, having electrodes sealed into it at $\mathrm{A}$ and $\mathrm{B}$. The tubes made use of are $30 \mathrm{~cm}$. long. By the use of these tubes not only is the liquid to a large extent protected from the air, but any gaseous products of electrolysis are at once apparent, and the different actions of the two electrodes can be well studied.

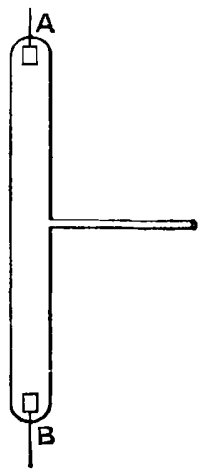

Freshly prepared, strong, diffusible arsenic sulphide solution was introduced into the tube on Saturday. The solution contained about 11 grams of the sulphide per litre. A battery with an electromotive force of about 10 volts was connected with the electrodes, and a galvanometer placed in the circuit. A deflection of $1.25^{\circ}$ was observed, corresponding, in the galvanometer used, to a current of about 0.000007 ampère.

On the actual experiment, a battery with electromotive force of 8 volts was used. At 2 P.M. on Saturday, the experiment was started. The negative pole was connected with $A$, the positive with $B$. The experiment was Ieft over Sunday, and on Monday at 10 A.M., the sulphide was found to have been driven down $11.5 \mathrm{~cm}$. By 1 P.M. it 
had been driven down $0.3 \mathrm{~cm}$. further, a total of $11.8 \mathrm{~cm}$. The rate of fall was now evidently becoming slower.

The repulsion being much greater from the negative pole, the current was now reversed at 1 p.m. to see whether the sulphide could be driven up again. $\mathrm{By} 4$ P.M., the level of the sulphide surface had risen $2 \mathrm{~cm}$. By Tuesday at 9 A.M., it had risen altogether 8.7 c.m. All this time there was absolutely no evolution of electrolytic gases. The battery was removed at 2.30 and again connected at 4.30 P.M. By Wednesday at 9 A.M., the total rise was $10 \cdot 2 \mathrm{~cm}$. It had now reached the positive electrode and the rate of rise which had at first been remarkably rapid, was now obviously much decreased. During Wednesday, the battery was connected up for $3 \frac{1}{2}$ hours without any further rise being observed, and on leaving it all night, the appearance remained exactly the same. The liquid round the negative pole was still coloured, thus showing a distinct difference from the action observed when the negative electrode was at the top.

On Thursday, the current was once more reversed. By Friday morning, the sulphide had been driven down $6 \cdot 2 \mathrm{~cm}$.

The liquid was now coagulated and tested for sulphuretted hydrogen and arsenious acid. No trace of either could be detected. One very small bubble of gas was now observed in the tube, the result of six days electrolysis.

Shellac.-Shellac does not dissolve in water, but it may be obtained in a state of pseudo-solution in that liquid by allowing a few drops of the alcoholic solution to fall into water. The shellac in this solution is repelled from the negative electrode.

Ferric Hydrate.-Some dilute colloidal ferric hydrate (dialysed) was similarly treated in a $\mathrm{V}$-tube, the electrodes being $30 \mathrm{~cm}$. apart. Left over night, the ferric hydrate was found to have been distinctly repelled from the positive electrode, the liquid in the corresponding limb of the tube being markedly paler in colour. At the same time, a very slight deposit of ferric hydrate was observed on the negative electrode.

Hoenoglobin.-Prure hæmoglobin crystals dissolved in water to a bright-red solution were treated in the same manner in a $U$-tube. The battery employed had an E.M.F. of about 8 volts. The electrodes were $13.5 \mathrm{~cm}$. apart. The experiment was left over night, the tube being immersed in ice to prevent any spontaneous decomposition of the hæmoglobin. The hæmoglobin was markedly repelled from the positive electrode. In the corresponding limb, $4 \mathrm{~cm}$. of colourless liquid was obtained, the hæmoglobin surface having sunk to that extent. At the same time, a small depression $(0.5 \mathrm{~cm}$.) had occurred in the opposite limb. The hæmoglobin thus repelled was examined spectroscopically. Throughout its extent it 
gave the normal absorption spectrum of oxyhæmoglobin. The apparently colourless liquid showed no absorption spectrum.

Magdala-Red.-In the case of Magdala-red, repulsion is observed from the positive electrode. With weak solutions. the liquid round the positive electrode becomes very nearly colourless. The experiments were performed in a $U$-tube. It was found that when a very strongly coloured solution was used, the Magdala-red obtained a certain concentration in the negative limb, and then no further action appeared to occur. With weak solutions, the experiment may be repeated many times without producing any change in the character of the Magdala-red. The colouring matter was dissolved in absolute alcohol, and the deflection of the galvanometer needle amounted in one case to about $0.25^{\circ}$. indicating a current of approximately 0.000001 ampere.

Several other liquids have been fonnd to exhibit similar properties, but further details are reserved for a future occasion, when we hope to have more thoroughly investigated the subject.

In the earlier stages of these experiments the suggestion was an obvious one, that the phenomena observed were due to a change in state of aggregation of the substance present in the solution. As the experiments multiplied, this explanation became increasingly improbable, but as it is the one which, at first sight, seems most natural, it is necessary to have a considerable weight of evidence before rejecting it. We shall, therefore, here shortly state some of the considerations which seem to us to conclusively prove the above theory to be untenable.

1. If we are to suppose that the downward movement of the dissolved substance is the result of mechanical settling, due to formation of larger aggregates, these aggregates must be of comparatively enormous size. This is obvious in the case of the arsenious sulphide solution above referred to, for while suspensions containing visible particles sometimes take many months to settle, the above solution at first consists of particles so small as to be readily diffusible, and yet under electrical infuence settles with great comparative rapidity. Moreover, these aggregates, if formed, must be again broken up on disconnecting the battery, since, then, not only does the downward movement cease, but the characteristic upward diffusion of the sulphide sets in. Now this subdivision of the larger aggregates is just one of the changes which, in the case of arsenious sulphide, we have found it impossible to effect. Thus arsenious sulphide $\beta$, in which we have been unable, as yet, to detect visiole particles, cannot, apparently, be converted into arsenious sulphide $\%$. Furthermore, a concentrated solution of arsenic $\gamma$ appears to contain somewhat larger aggregates than those of a dilute solution, though they are still quite 
invisible. On diluting the strong solution, these do not seem to be broken up, for a solution so dilnted is distinctly more fluorescent than a dilute solution directly obtained.

2. The upward movement of the sulphide, in the manner described, is similarly opposed to the theory, but it has been suggested that this is due to the removal of the upper surface of the liquid from the sphere of action of the negative electrode, consequent breaking up of the aggregates, and resulting liability to diffusion. Apart from previous considerations, this is opposed by the fact that the upward movenent observed is entirely different, both in rapidity and character, from the ordinary diffusion of the sulphide. Thus the rise of level of $2 \mathrm{~cm}$. in three hours, already described, would probably have occupied more than 24 hours in the case of simple diffusion. Moreover, in that case the sharply-defined surface of the concentrated solution steadily rose, with scarcely any of the shading off at the. surface observed in ordinary diffusion.

3. That no formation of larger aggregates occurs in the case of Magdala-red, is plain from the following experiment. In a suitable apparatus, with electrodes about $2 \mathrm{~cm}$. apart, the solution was treated electrically, while a ray of lime-light was passed between the electrodes. No polarisation of the scattered light could be observed. It is therefore obvious that no marked condensation had occurred.

In the case of Magdala-red, also, the concentration of the solution may be made to occur towards the upper or lower electrodes, according to the arrangement of the connections with the battery.

4. The remaining hypothesis, that the apparent repulsion may be due to decolorisation produced by the products of electrolysis, is obviously not in accord with the results obtained with arsenious sulphide. Indeed, when we consider how excessively small an amount of such products there must be (as deduced from the observed strength of current), we see that this hypothesis scarcely needs refutation. Obviously, it cannot be applied to such a case as that of shellac, and, to cite another instance, the beliaviour of oxyhæmoglobin also disproves it. If decomposed, as suggested, it would not yield colourless products, but would show the spectrum of hæmatin.

5. Microscopical examination, so far as at present conducted, is in accord with the above arguments. An indigo suspension, in which the particles were well seen with a $1 / 5$ th objective, showed us these particles repelled rapidly from one electrode and attracted towards the other without any apparent change in the state of aggregation. Under similar influence, arsenic $\gamma$ showed no visible particles, and, though we have not been yet able to examine the arsenic with a higher power, one would certainly expect particles capable of settling at the above described rate to be visible with the power employed. 
It may be that the aggregates in these solutions are in an electrified condition, bat on this subject it would be rash to dogmatise at present.

The only phenomenon in any way resembling the above with which we are acquainted is that, on passing a current through acidified water divided into two parts by a septum, the level of the water rises on that side of the septum at which the negative electrode is immersed (Porret). How far this phenomenon is really analogous to the above, we cannot say at present with any certainty.

\section{Theoreticat.}

It seems to us that in the preceding part of this paper, we have made out at least a good primâ facie case for the belief that between obvious suspension and crystallisable solution there is no break in the series of grades of solution. We have seen that colloidal solution in many ways resembles liquid containing solid particles in suspension. We have seen that we can pass from solation in which these particles are visible under the microscope to those in which they are invisible, but indiffusible, and thence to those invisible and diffusible. These solutions show also gradations of permanency, and differ as to re-solubility. In some cases, such as arsenic sulphide, we cannot redissolve the solid after precipitation; in others, mercuric sulphide, starch, for example, we can readily effect re-solntion. We are thus presented with varying degrees of action between the solvent and the solid. Further up the series we find evidence, as in silicic acid, of colloids in a still finer state of subdivision, and giving no positive evidence of the presence of solid particles. We then come to crystalloids, such as the compounds $9 \mathrm{Fe}_{2} \mathrm{O}_{3}, \mathrm{FeCl}_{3}$ and hæmoglobin, showing evidence of particles, yet by their ready re-solubility, permanence, and power to resist the influence of salts are evidently in a state of comparatively perfect solution. From these we pass to crystalloids in which no particles are discernible, but which are not electrolytes, and finally to those which are electrolytes. True fluorescent solutions show a luminous beam when a ray of light is passed through them, but the scattered light is not polarised. This kind of fluorescence is apparently unconnected with molecular complexity.

The electrical evidence is also of great interest from the point of view of the general theory of solation. We hope before long to present to the Society a further series of results on this subject, but we may here point out the similarity of behaviour exhibited, under this test, by more and less complex molecules. Colloidal arsenic sulphide is repelled from one of the electrodes in a similar manner to

vOL. LXI. 
colloidal ferric hydrate, to hæmoglobin, and to Magdala-red. What the precise differences are in the different cases and in the case of electrolytes must be examined into on another occasion. We are here only concerned to point out the similarities.

From obvious suspension, then, we pass to colloidal solution, from colloidal solution to non-electrolytic crystallisable solution, and from these it is probably only one step further to electrolytic solutions, now often regarded as dissociated. This change may be regarded as continuous, and there is no reason to suppose that a sharp line separates the different states of matter from each other. The tendency of all modern investigation is to show that there are no such things as boundaries in nature, and in this case, like others, they seem to disappear on examination.

But it is obviously not a question of mere subdivision, nor can we regard the colloidal solution as cases of mere mechanical suspension. At one end of the series, where a powdered solid is kept temporarily suspended by being thoroughly shaken. up with a liquid, there seems to be absolutely no action, except those of a purely mechanical kind, between the solid and the liquid wherein it is suspended. As, however, we pass up the series to matter in a more perfect state of suspension, it becomes impossible to avoid assuming some action, however slight and indefinite, of a more chemical nature between the liquid and the solid, which it retains in suspension. For instauce, the fact that sulphuretted hydrogen water will split up precipitated mercuric sulphide into a state of subdivision so fine as to closely resemble ordinary colloidal solution appears inexplicable on a purely mechanical hypothesis. Some attraction, in its nature more resembling that of chemical affinity, there must surely be between water. molecules or sulphuretted hydrogen molecules, or both, and the particles of mercuric sulphide. Passing further up the series, we may take as another example, the case of soluble starch. This substance dissolves without difficulty in water, but, as long suspected, and as further proved by our experiments, the starch exists in the solution in the form of finely-divided particles, and these are not in too fine a state of subdivision to be revealed by a ray of light. Such a result seems inexplicable, unless we assume something more nearly approaching chemical attraction between water and starch.

In the case of a solution of ferric hydrate in ferric chloride, there is no doubt whatever that a chemical attraction exists between solvent and solid. Not only is a definite crystalline compound of ferric oxide and chloride said to have been isolated, but, on coagnlating a dialysed solution, which, as is well known, retains no inconsiderable amount of chloride, the chloride is found to be thrown out of solution along with the hydrate. But, with a large excess of chloride present, this 
solution shows a well-marked, luminous beam, the light of which is completely polarised. The solution appears to be perfectly permanent. The question naturally arises, In what manner is the ferric chloride in the solution combined with the hydrate? Are the aggregates revealed by a ray of light composed of the two components of the compound, or is the aggregate of ferric hydrate loosely combined with, or attracted by, the surrounding molecules of ferric cbloride which are in more perfect solution? It is difficult to decide such a question; but it seems to us that probably both forms of combination occur at once. In the first case, we should have an example of mere mechanical suspension, unless we made the further supposition that the combined aggregates were loosely attracted by the water molecules in their neighbourhood. But the water molecules do not seem to exert so powerful an influence, for, on very prolonged dialysis and consequent gradual dissociation of the ferric chloride hydrate compound, the solution, as is well known, has a tendency to gelatinise on keeping. The simplest supposition appears, therefore, to be that the aggregates of ferric hydrate are held in solution almost entirely by their loose union with surrounding molecules of ferric chloride, themselves in a state of more perfect solution. The fact that it is not merely the more stably combined ferric chloride which assists solution is proved by the experiment of adding calcic chloride to the dialysed and undialysed liquid. In the former, precipitation is rapid; in the latter, it is far more difficult to produce. It certainly seems illogical to regard a solution of this kind as a case of mere mechanical suspension.

It may be remembered that the cases of certain sulphides soluble in sulphuretted hydrogen water present analogies to the present one. These sulphides (as shown in the paper "On some Metallic Hydrosulphides," p. 114) combine with a certain amount of sulphuretted hydrogen in the liquid in which they dissolve. The compound thus formed has, as a rule (see Mercuric Sulphide), a very complicated structure, and, in the case of these solutions, is often very unstable. On coagulating such a solution, however, the combined sulphuretted hydrogen is thrown out with the precipitate. A mercuric sulphide solution, when coagulated with calcic chloride, evolves no sulphuretted hydrogen (see also Copper). These cases entirely support the present view of colloidal solution. At the same time, the influence of salts in producing coagulation is difficult to explain.

The cases of albumen and of hæmoglobin are analogous. Albumens dried at $40^{\circ}$ are re-soluble in water, and we must suppose a certain chemical attraction between solvent and solid. Hæmoglobin may be easily recrystallised and redissolved. Yet its solution reveals particles under the scrutiny of the beam. 
It may be suggested that all that happens in these cases is a separation of these aggregates or molecules from the mass by virtue of their own vibratory motion, and that these small masses are able to support themselves in a liquid medium. Against this view must be set the non-diffusibility of some of the solutions such as starch. Besides this, such a theory is quite inadequate to account for the entirely different behaviour of different solvents towards the same solid. Starch, for instance, is not soluble in alcohol. Finally, in a case of mere mechanical suspension of very fine particles, must it not be supposed that in a very dilute suspension of this kind the particles would at least tend to settle with comparative rapidity to a concentration, at which the collisions with the lower vibrating particles would nearly counterbalance the tendency to further descent? Yet in many such dilute suspensions no such settling is observed. This may be partly accounted for by supposing the particles to be exceedingly minute; but in order to meet the requirements, a minuteness so extreme must be supposed (see Introduction, p. 150), that it is simpler to concede molecular attraction at once.

What, then, is the most rational way of regarding these phenomena of pseudo-solution and molecular solution? It seems to us by far the most simple course to suppose that in every case of so-called very fine suspension there is a certain degree of molecular attraction between the suspended solid and the solvent. The case of shellac affords a well-known example. According as this attraction is less or greater, the solution will be less or more permanent. Moreover, it is obvious that upon this attraction and that of the aggregates for each other, will depend the re-solubility of the solid. Shellac is not re-soluble. The water molecules do not attract the shellac aggregates with sufficient force to shake them asunder. On the other hand, in other cases, resolution is aided by mechanical agitation or boiling, \&c., all of which favour the breaking up of the solid.

In cases where resolution is more readily effected (mercuric sulphide, albumen, hæmoglobin), either the particles of the solid are held together less firmly, or the attraction of the water molecules is greater. That the subdivision should proceed no farther than it does is easily accounted for by supposing, what is extremely probable, that the molecules of a solid are bound together in multiple aggregates which become looser with greater aggregation. These can be easily shaken asunder into their component aggregates of less complexity. But here the forces at work may be found powerless to produce further change. The less complex aggregates are bound together too firmly to be further dissociated, and. under certain circumstances, may be found acting as wholes. This explains why, in dissolving ferric hydrate in ferric chloride, the subdivision proceeds no farther than it 
does, and why a substance so readily soluble in water as neutral "Congo-red" yet reveals the presence of particles in its solution. Tt may here be noted, in support of these views, that gradual condensation to form aggregates of greater complexity may be observed in some cases. Thus colloidul antimony sulphide when freed from tartrate, shows a gradual and continuous condensation from aggregates quite invisible, through stages of increasing fluorescence, to aggregates visible under the microscope. The progress of a similar condensation is indicated by increasing fluoressence in antimony sulphide solutions after keeping for some months. Silicic acid presents us with a case of similar regular condensation. In the case of chromic chloride, we have probably an example of the gradual breaking up of aggregates through the various stages of pseudosolntion till crystallisable solution is arrived at. The precise analogy between this and that of antimony sulphide in the reverse direction makes us think it probable that we are here following a continuous breaking up of molecular aggregates.

It may be observed that, if these views are correct, we have here an interesting analogy between the lower and ligher grades of solntion. For, whilst in the lower grades we have combination between very large aggregates of the dissolved substance and the water molecules in their neighbourhood, it has been shown with great probability by Pickering and others that in the case of some of the higher grades of solution the molecules of the dissolved substance combine with a large number of water molecules to form very high hydrates. This does not seem to us to affect the question of probable dissociation into ions in the most perfect solution.

Throughout the above argument we have necessarily been assuming, what must now be fully stated, that these fine particles revealed by the luminous beam are simply large molecular aggregates, and retain many of their molecular properties. As wholes they can enter into loose combination, and as wholes they can attract each other with gradual formation of larger aggregates.

These molecular aggregates may even attain a complexity so great that they become visible under the microscope, and yet do not entirely lose their molecular properties (mercuric sulphide). What then is the interpretation of the continuous vibratory movement of these aggregates as seen under the microscope? Enormous as must in all probability be the number of molecules associated to form a visible aggregate, yet as the transition from them to states of undoubted molecular subdivision seems perfectly continuous, we can scarcely avoid the conclusion that this vibratory motion is one of the molecular properties still retained by them, and that what we are 
here observing is simply molecular vibration. On the other hand, it might be conceived that these oscillations are due to the impacts of the liquid molecules. On the whole this seems less probable, for considering the relative sizes of the particles and the water molecules, it seems doubtful whether these impacts would occur with sufficient irregularity to cause visible motion. Furthermore, there is no proof of the existence of large molecular aggregates of water which would be competent to produce such effect. The conclusion that we are here actually observing molecular vibration seems a rash one, but it appears at present to be in accordance with facts, and to offer the most rational explanation of Brownian movement.

As to the electrical portion of our work, we shall not at present offer any theory, though the subject is an obviously tempting one for speculation. But on this point, and on the general question discussed in this paper, we hope in a short time to have more results to communicate.

Our main conclusion, therefore, is that from first to last, from pseudo-solution to crystalloidal solution, we are concerned with chemical as well as with physical forces, and that the progress from the lower to the higher terms of the series is acconipanied by increasing fineness of subdivision of the dissolved matter, and by increasing definiteness in the action of the chemical forces.

\section{Summary.}

We shall here briefly review in outline the main results of this and the preceding paper.

I. Experimental.-1. The following inorganic colloidal solutions reveal particles visible under the microscope :-

Mercuric sulphide, arsenious sulphide ( $\alpha$ ).

2. The following inorganic colloidal solutions contain the dissolved substances in states of aggregation recognisable by optical or other means :-

All colloidal sulphide solutions.

Ferric hydrate, chromic hydrate, aluminium hydrate, silicic acid.

3. Owing to the difficulty of preparing organic colloids in a state of purity, only the following have, as yet, been examined. They all exhibit a state of subdivision not too fine to be recognised by the methods employed :-

Cellulose, starch, Congo-red (acid and neutral).

[Alkaline Congo-red is in a state of much more perfect solution. 7 
4. The following colloids exist in solution in a state of subdivision too fine for the aggregates to be detected by ordinary methods:-

Molybdic acid, silicic acid (in presence of hydrochloric acid).

5. The following crystallisable solutions reveal molecular aggregates large enough to be detected by optical or other methods :-

Ferric hydrate in ferric chloride (said to crystallise as $9 \mathrm{~F}_{2} \mathrm{O}_{3}, \mathrm{FeCl}_{3}$ ).

Oxyhæmoglobin, carbonic oxide hæmoglobin.

6. Silicic acid, after dialysis, begins to exhibit signs of advancing molecular condensation. This is analogous to the case of antimony sulphide, in which the steady molecular condensation is under certain circumstances perfectly demonstrable (see above).

Chromic chloride, when first dissolved, appears to exist in the form of large aggregates, which are gradually broken up with final formation of crystallisable solution.

II. Theoretical. -We consider that we have made out a good primád facie case for the belief that there is a continuous series of grades of solution passing without break from suspension to crystallisable solution. In the lowest grades of solution, a certain loose attraction exists between the "suspended" particles and the molecules of the solvent. This conclusion is in accordance with the experimental evidence. The very finely divided particles in the lower grades of solution are simply large molecular aggregates retaining many of their molecular properties. In passing up the series through the different grades of solution, these aggregates on the whole become smaller, or, at least, consist of a smaller number of molecules, and the forces by which they are held in solution become more definitely those of chemical attraction.

III. Electrical Behaviour of certain Solutions.-Mention bas been made in the paper of a new property of certain solutions. It seems to hold with a wide range of solutions extending from suspension to crystalloidal solution. This property consists in the repulsion of the dissolved substance as an unaltered whole from one of the electrodes of a battery if these be immersed in the solution. In the case of colloidal arsenic sulphide, the sulphide aggregates are repelled from the negative electrode. They are also repelled, but much less strongly, from the positive electrode. Crystalloidal Magdala-red dissolved in absolute alcohol is repelled from the positive electrode. In this case there seems to be no repulsion from the negative. This property is of much interest in itself, and may prove of much value in connecting the higher grades of solution.

Researches on the above subjects are now in progress. 
In conclusion, we wish to express our hearty thanks to Professor. Ramsay, who has most kindly encouraged and advised us throughout the whole of the work of the three preceding papers. It is with sincere pleasure we acknowledge that without his kind help it would have been difficult to bring these researches to a satisfactory issue.

We should like also, among others, to thank Professor Carey Foster for kind assistance in the electrical portion of our work, and Professor E. A. Schäfer for placing his microscope at our disposal.

University College,

London. 